\title{
Прыжковая проводимость и диэлектрическая релаксация в барьерах Шоттки на основе GaN
}

\author{
() Н.И. Бочкарева ${ }^{1}$, В.В. Вороненков ${ }^{1}$, Р.И. Горбунов ${ }^{1}$, М.В. Вирко ${ }^{2}$, В.С. Коготков ${ }^{2}$, А.А. Леонидов ${ }^{2}$, \\ П.Н. Воронцов-Вельяминов ${ }^{3}$, И.А. Шеремет ${ }^{4}$, Ю.Г. Шретер ${ }^{1, \uparrow}$ \\ ${ }^{1}$ Физико-технический институт им. А.Ф. Иоффе Российской академии наук, \\ 194021 Санкт-Петербург, Россия \\ ${ }^{2}$ Санкт-Петербургский политехнический университет Петра Великого, \\ 195251 Санкт-Петербург, Россия \\ ${ }^{3}$ Санкт-Петербургский государственный университет, \\ 199034 Санкт-Петербург, Россия \\ ${ }^{4}$ Финансовый университет при Правительстве Российской Федерации, \\ 125993 Москва, Россия \\ ๑ E-mail: y.shreter@mail.ioffe.ru
}

(Получена 24 января 2017 г. Принята к печати 9 февраля 2017 г.)

Представлены результаты исследования зависимостей тока и емкости от прямого смещения диодов Шоттки $\mathrm{Au} / n-\mathrm{GaN}$, а также спектров подзонного оптического поглощения и дефектной фотолюминесценции объемных кристаллов и тонких слоев $n$-GaN. Показано, что туннелирование с участием дефектов является доминирующим механизмом транспорта при прямых смещениях контактов Шоттки на $n$-GaN. Зависимости тока и емкости от прямого смещения отражают энергетический спектр дефектов в запрещенной зоне $n$-GaN: рост с энергией плотности глубоких состояний, ответственных за желтую фотолюминесценцию в GaN, и резкий экспоненциальный хвост состояний с урбаховской энергией $E_{\mathrm{U}}=50$ мэВ вблизи края зоны проводимости. Уменьшение частоты перескоков электронов вблизи интерфейса $\mathrm{Au} / n-\mathrm{GaN}$ приводит к широкому распределению локальных времен диэлектрической релаксации и изменению распределения электрического поля в области пространственного заряда при прямых смещениях.

DOI: 10.21883/FTP.2017.09.44888.8528

\section{1. Введение}

Значительный прогресс в развитии силовой электроники на $\mathrm{GaN}$ достигнут в последнее десятилетие. На массовый рынок вышли мощные быстродействующие транзисторы на основе $\mathrm{GaN}$, превосходящие кремниевые аналоги по рабочим частотам и плотности мощности [1]. В результате длительной оптимизации кремниевых приборов, физические свойства материала все больше становятся лимитирующим фактором для их дальнейшего совершенствования. В то же время параметры приборов на основе $\mathrm{GaN}$ еще далеки до своего теоретического предела, определяемого большим электрическим полем пробоя, высокой подвижностью и насыщенной дрейфовой скоростью электронов, хорошей теплопроводностью GaN. Реализация этого потенциала в большой степени связана с необходимостью понимания роли высокой плотности протяженных и точечных дефектов в нитридах III группы в транспорте носителей через потенциальные барьеры и механизма влияния дефектов на параметры приборов.

Ключевым элементом многих силовых и СВЧ приборов на основе $\mathrm{GaN}$ являются контакты Шоттки. Реализация контактов Шоттки на $\mathrm{GaN}$ с высоким потенциальным барьером, малыми токами утечки и высокой плотностью максимального прямого тока часто является основной технологической проблемой. Избыточные токи утечки в $\mathrm{AlGaN} / \mathrm{GaN}$-барьерах Шоттки, используемых в качестве затвора в НЕМТ-транзисторах, ведут к потерям мощности, нестабильности характеристик транзистора и шумам тока канала [2,3], уменьшают надежность работы. Эффект токового коллапса, связываемый с захватом электронов в области затвора Шоттки, также ограничивает максимально достижимую мощность НЕМТ-транзисторов.

Механизм транспорта в контактах Шоттки на GaN часто обсуждается в литературе, по аналогии с $\mathrm{Si}$, в рамках моделей термоэлектронной, полевой или термополевой эмиссии [4,5]. Для объяснения причин больших токов утечки привлекаются и развиваются известные модели туннелирования с участием дефектов, предложенные ранее для избыточных туннельных токов в $\mathrm{Si}$ или в тонких слоях $\mathrm{SiO}_{2}$ : многоступенчатое туннелирование через серию дискретных уровней [6], туннелирование с участием дефектных состояний, однородно распределенных по энергии в запрещенной зоне [7], многофононное туннелирование $[8,9]$, прыжковая проводимость с переменной длиной прыжка по дислокациям [10]. В качестве возможных причин утечек рассматриваются также неоднородность высоты барьера Шоттки по площади диода [11] и неоднородность легирования по глубине области пространственного заряда (ОПЗ), вызванная непреднамеренным образованием доноров в приповерхностном слое $\mathrm{GaN}$ [2]. В работе [12] предложена модель прыжкового транспорта через ОПЗ $p$-n-структур с квантовыми ямами InGaN/GaN по состояниям центров окраски, образующих глубокие экспоненциальные хвосты плотности состояний в $\mathrm{GaN}$ и ответственных 
за полосы дефектной фотолюминесценции в видимой и ближней УФ областях спектра.

Цель данной работы - рассмотреть влияние ловушек на туннелирование электронов через область пространственного заряда барьеров Шоттки в GaN. B частности, рассмотреть их влияние на проводимость и время диэлектрической релаксации при прямых смещениях, а также указать характерные проявления этого механизма транспорта электронов в $I-V$ и $C-V$ характеристиках диодов.

\section{2. Эксперимент}

Диоды Шоттки изготавливались на основе слоев $\mathrm{GaN}$ $n$-типа проводимости, выращенных на сапфировых подложках с ориентацией ростовой поверхности (0001) методом газофазной хлорид-гидридной эпитаксии (HVPE) (диоды А и В) [13] и эпитаксии из металлоорганических соединений (MOCVD) (диоды C и D). Толщина слоев $\mathrm{GaN}$ в диодах $\mathrm{A}, \mathrm{B}, \mathrm{C}, \mathrm{D}$ составила $d=40,20,1.5$ и 1.5 мкм, концентрация электронов $2 \cdot 10^{18}, 2 \cdot 10^{17}$, $3 \cdot 10^{17}$ и $3 \cdot 10^{17}$ соответственно. Контакты Шоттки площадью $10^{-3} \mathrm{~cm}^{2}$ в диодах А и В и $10^{-4} \mathrm{~cm}^{2}$ в диодах С и $\mathrm{D}$ создавались термическим напылением Au в вакууме. Омические контакты на основе In изготавливались на поверхности базового слоя $n$-GaN.

Измерения статических вольт-амперных характеристик диодов проведены с помощью Keithley 238, емкостные измерения проведены на частоте 1 МГц с помощью CV анализатора Keithley 590. Измерения фотолюминесценции эпитаксиальных слоев $n-\mathrm{GaN}$, а также объемных кристаллов $\mathrm{GaN}$ толщиной $d=200$ мкм [13], выращенных методом HVPE, проводились при комнатной температуре с помощью спектрометра Avantes. Фотолюминесценция возбуждалась $\mathrm{He}-\mathrm{Cd}$-лазером на длине волны $\lambda=325$ нм со стороны поверхности роста и детектировалась „на просвет“ со стороны подложки. Интенсивность возбуждения составляла $1 \mathrm{BT} / \mathrm{cm}^{2}$. Измерения спектров оптического поглощения проведены методом оптического пропускания с использованием в качестве источника излучения вольфрамовой лампы накаливания (ОИ-24). Спектры падающего на кристалл и прошедшего через кристалл излучения измерялись с помощью спектрометра Avantes.

\section{3. Экспериментальные результаты}

\section{1. Зависимости плотности тока и емкости от прямого смещения}

На рис. 1, а представлены зависимости плотности прямого тока $j$ диодов Шоттки А, B, С и D от приложенного прямого напряжения $j(V)$ (кривые $1^{\prime}-4^{\prime}$ ) и от падения напряжения на области пространственного заряда контакта Шоттки $j\left(V_{j}\right)$ (кривые $1-4$ ), где $V_{j}=V-I \cdot R_{s}$, $R_{s}$ - последовательное сопротивление диода, $I$ - ток.
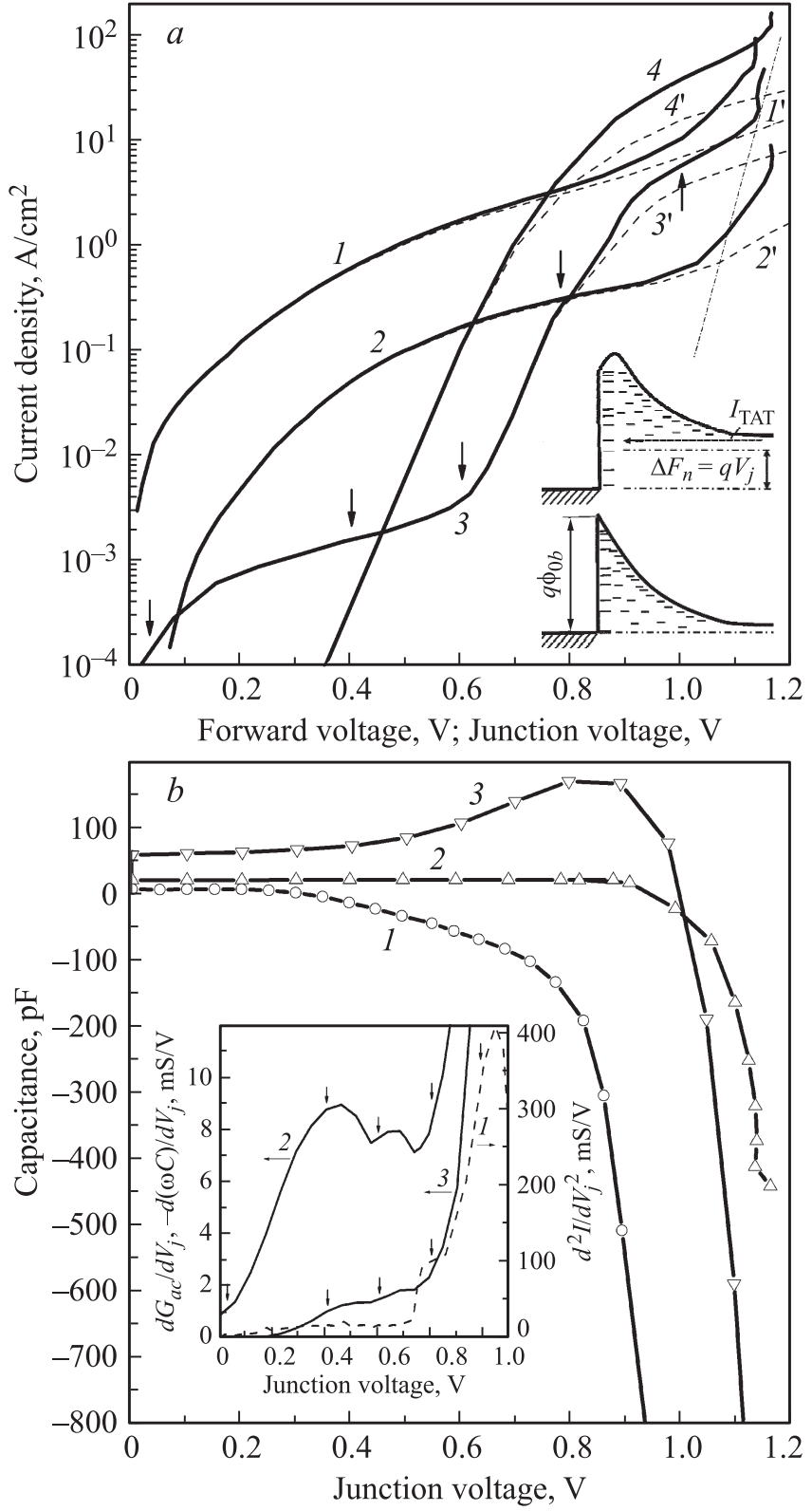

Рис. 1. $a-$ зависимости плотности прямого тока от приложенного прямого напряжения $j(V)\left(1^{\prime}-4^{\prime}\right)$ и от напряжения на области пространственного заряда $j\left(V_{j}\right)(1-4)$ для диодов Шоттки А $\left(1,1^{\prime}\right)$, В $\left(2,2^{\prime}\right), \mathrm{C}\left(3,3^{\prime}\right)$ и $\mathrm{D}\left(4,4^{\prime}\right)$. Стрелками на кривых $\lg j\left(V_{j}\right)$ диода С отмечены пороговые напряжения $V_{\text {th }}$, вблизи которых наблюдается уменьшение фактора идеальности кривых $\lg j\left(V_{j}\right)$. Штрихпунктирной линией показана аппроксимация зависимости надбарьерного термоэмиссионного тока, характеризующегося фактором идеальности $n=1$, от прямого смещения. На вставке показан профиль зоны проводимости для барьера Шоттки с прыжковой проводимостью в области пространственного заряда в равновесии (внизу) и при прямом смещении (наверху). $b$ - зависимости емкости от прямого напряжения на области пространственного заряда для диодов Шоттки А (1), В (2) и С (3). Частота измерений 1 МГц. На вставке - зависимости $d^{2} I / d V^{2}(1), d G_{\text {ас }} / d V_{j}(2)$ и $-d(\omega C) / d V_{j}$ (3) от прямого напряжения на области пространственного заряда для диода А. Стрелками отмечены величины пороговых напряжений $V_{\text {th }}$ из кривой $\lg j\left(V_{j}\right)$ диода $\mathrm{C}$ (рис. $1, a$, кривая 3$)$. 
Прямые $j-V_{j}$-характеристики имеют типичный для $\mathrm{GaN}$-диодов Шоттки вид, который обычно описывается в литературе известной эмпирической формулой:

$$
I=I_{0} \exp \left(q V_{j} / n k T\right)
$$

где параметр $n\left(V_{j}\right)$ - фактор идеальности, $k-$ постоянная Больцмана, $T$ - абсолютная температура, $I_{0}-$ предэкспонента. По аналогии с $\mathrm{Si}$, по величине фактора идеальности $n\left(V_{j}\right)$ принято судить о преобладающем механизме тока, протекающего через барьер: термоэмиссионного при $n=1$, рекомбинационного при $n=2$ и туннельного при $n>2$. Плечо на $I-V$-кривых в области малых напряжений обычно связывается с туннельной утечкой тока, увеличение наклона $I-V$-кривой в области средних напряжений - с доминированием надбарьерного термоэмиссионного тока, последующее уменьшение наклона с ростом напряжения - с падением напряжения на последовательном сопротивлении диода. Однако поведение тока с ростом напряжения на ОПЗ контакта Шоттки и $j\left(V_{j}\right)$-характеристики исследуемых диодов противоречат этим представлениям.

Как можно видеть из рис. $1, a, \lg j\left(V_{j}\right)$-характеристика диода $\mathrm{C}$ имеет ступенчатый вид с пороговыми напряжениями вблизи $V_{\text {th }}=0,0.6$ и $0.9-1 \mathrm{~B}$, небольшие ступени есть также вблизи $V_{\text {th }}=0.4$ и 0.8 В. В области $V=0.6-0.9$ В наблюдается рост тока на 3 порядка, и при плотностях тока $j=0.01-0.1 \mathrm{~A} / \mathrm{cm}^{2}$ фактор идеальности $n\left(V_{j}\right)$ близок к $n=1$. При $V_{j}>1.1 \mathrm{~B}$ также наблюдается более резкий рост тока с напряжением $V_{j}$, характеризующийся фактором идеальности $n \leq 1$. Резкий рост тока при $V_{j}>1.1$ В наблюдается и у диодов $\mathrm{A}$, $\mathrm{B}$ и D. Ток у диодов А и В также сильно растет в области $V_{j}=0-0.6 \mathrm{~B}$, при $V_{j}>0.6 \mathrm{~B}$ наблюдается уменьшение фактора идеальности $n\left(V_{j}\right)$.

Из представленных на рис. $1, b$ зависимостей дифференциальной емкости диодов $\mathrm{A}, \mathrm{B}$ и $\mathrm{C}$ от прямого смещения $V_{j}$, видно, что у всех диодов при увеличении $V_{j}$ наблюдается отрицательная емкость, отражающая инерционное возрастание проводимости [12]. На вставке к рис. 1, $b$ представлены зависимости производных кривых статической дифференциальной проводимости $d G_{\mathrm{dc}} / d V_{j}=d^{2} I / d V^{2}$ (кривая 1 ), активной $d G_{\mathrm{ac}} / d V_{j}$ (кривая 2) и реактивной, $-d(\omega C) / d V_{j}$ (кривая 3) проводимости на частоте $1 \mathrm{MГц} \mathrm{от} \mathrm{прямого} \mathrm{смещения} V_{j}$ для диода А. Из рис. $1, a$ и $b$ видно, что „Горбы“ и максимумы на этих кривых наблюдаются вблизи тех же пороговых напряжений, что и на кривой $\lg j\left(V_{j}\right)$ диода С.

Высота барьера Шоттки $q \varphi_{0 b}$, определенная с помощью аппроксимации участков $j-V_{j}$ и $j-V$ характеристик диодов $\mathrm{A}-\mathrm{D}$, при больших плотностях тока составила $q \varphi_{0 b}=1.08-1.12 \mathrm{eV}$, что хорошо согласуется с литературными данными для высоты барьера Шоттки $\mathrm{Au} / n-\mathrm{GaN}$ [14-16]. Если связать резкий рост тока вблизи напряжения плоских зон с протеканием надбарьерного термоэмиссионного тока, то, как можно видеть из pис. 1, $a$, ток диода $\mathrm{C}$ в области средних напряжений более чем на 3 порядка превышает надбарьерный термоэмиссионный ток. Это указывает на подбарьерный механизм протекания тока, характеризующийся фактором идеальности, близким к $n=1$.

\section{2. Подзонное оптическое поглощение в слоях GaN}

Информацию об энергетическом спектре локализованных состояний могут дать измерения спектров подзонного оптического поглощения.

На рис. $2, a$ и $b$ представлены спектры оптической плотности $\alpha d=\ln \left(\Phi_{0} / \Phi_{\tau}\right)$, где $\Phi_{0}$ и $\Phi_{\tau}-$ интенсивности падающего и прошедшего света соответственно и спектры фотолюминесценции объемного кристалла $\mathrm{GaN}$ толщиной $d=200$ мкм, выращенного методом HVPE (кривые 1), и слоев $n$-GaN, выращенных методами HVPE и MOCVD толщиной $d=40$ и 1.5 мкм соответственно (кривые 2 и 3 ).

В спектре фотолюминесценции объемного кристалла наблюдаются характерные для $\mathrm{GaN}$ полосы красной (RL) (пиковая энергия $h v_{\mathrm{RL}}=1.85$ эВ), желтой $(\mathrm{YL})\left(h v_{\mathrm{YL}}=2.2{ }^{\ni} \mathrm{B}\right)$, голубой $(\mathrm{BL})\left(h v_{\mathrm{BL}}=2.9 \ni \mathrm{\jmath}\right)$, ИК-фотолюминесценции (IRL) в области $h v<1.3$ эВ и близкраевой УФ фотолюминесценции в области $3.4>h v_{\text {UVL }}>3.1$ эB [17]. Спектры фотолюминесценции слоев $n$-GaN содержат те же полосы фотолюминесценции, что и спектр объемного кристалла $\mathrm{GaN}$.

В спектре оптической плотности объемного кристалла $\mathrm{GaN}$ (рис. 2, $a$, кривая 1) видно резкое увеличение поглощения вблизи оптических пороговых энергий фотонов $h v_{\text {th }}=2.3,3$ и 3.2 эВ. Спектры оптической плотности тонких слоев $n$-GaN (рис. 2, $a$, кривые 2 и 3 ) более сглажены, тем не менее ступенчатый рост поглощения в спектральной области $3.4>h v>2.3$ эВ виден.

Резкое увеличение оптического поглощения в $\mathrm{GaN}$ вблизи оптических пороговых энергий $h v_{\text {th }}=1.1-1.3$, $2.1-2.5,3.1-3.2$ эВ наблюдалось ранее в ряде работ [18-21]. В работе [18] показано, что оптическое поглощение увеличивается с энергией фотона $h v$ как $\exp \left(h v / E_{0}\right)$, с параметром $E_{0}$, изменяющимся в области от 1.5 до 3 эВ от 180 до 280 мэВ и равным 50 мэВ в урбаховских хвостах плотности состояний в области $\sim 3.10-3.42$ эВ. Исследование спектров подзонного оптического поглощения в объемных кристаллах $\mathrm{GaN}$ в работе [12] позволило сделать вывод, что спектры поглощения представляют собой суперпозицию полос поглощения, обусловленных внутрицентровыми переходами в центрах окраски, ответственных за полосы фотолюминесценции в $\mathrm{GaN}$. Полосы поглощения сдвинуты в коротковолновую область спектра относительно полос фотолюминесценции. Используя известную из литературы идентификацию дефектов, ответственных за полосы фотолюминесценции в $\mathrm{GaN}$ [17], можно связать рост поглощения в спектральных областях $2.6>h v>2.3$ эВ и $3.1>h v>2.9$ эВ с комплексами кислорода в азотных 

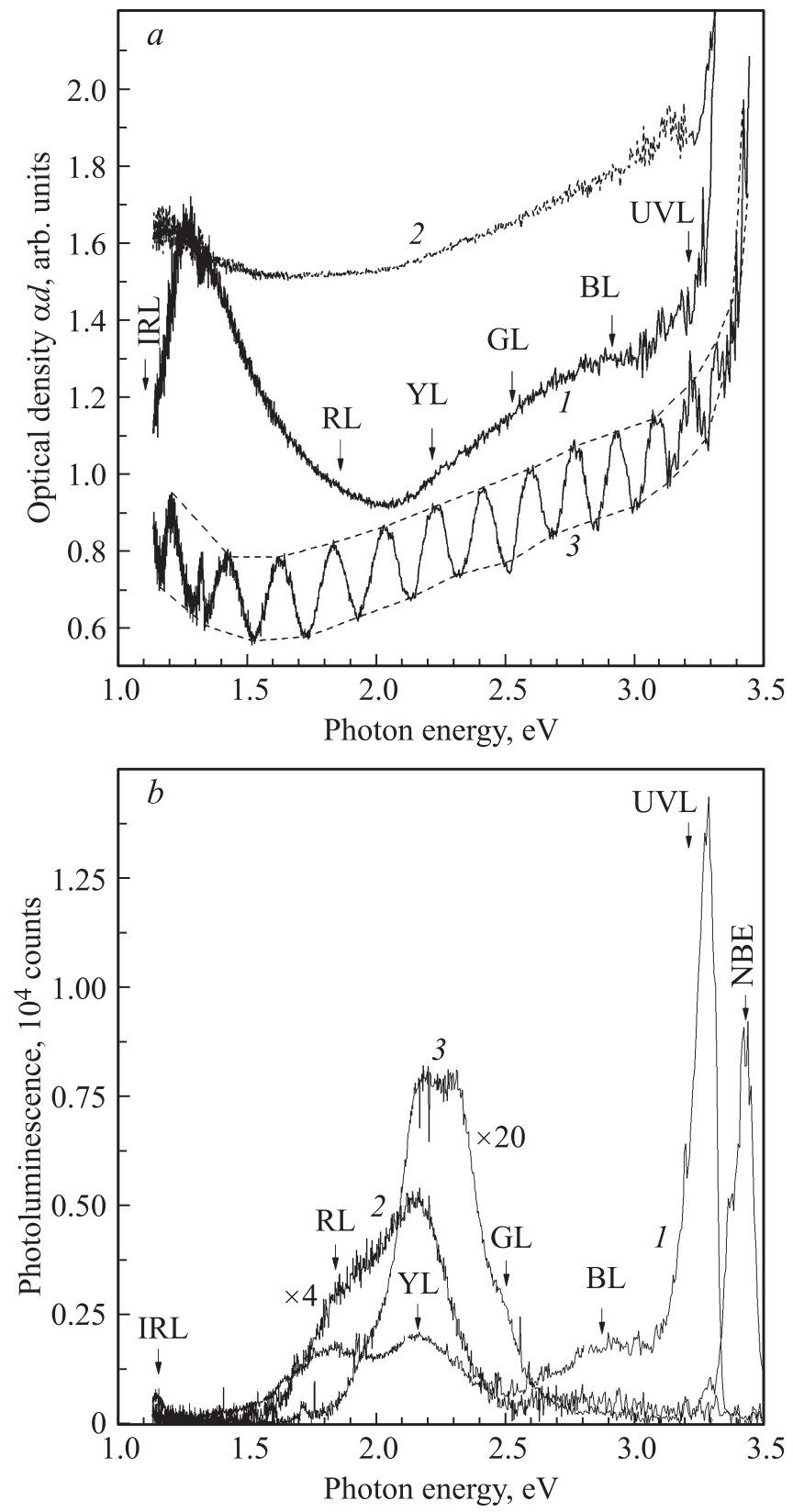

Рис. 2. Спектры оптического поглощения $(a)$ и фотолюминесценции, возбуждаемой Не-Сd-лазером $(\lambda=325$ нм $)(b)$, объемного кристалла $n$-GaN (1) и слоев $n$-GaN, выращенных методом HVPE (2) и MOCVD (3), $d$, мкм: $1-200,2-40$, $3-1.5$. Пунктирными линиями показаны нижняя и верхняя огибающие спектра поглощения (3). Стрелками отмечены спектральные положения максимумов интенсивности основных полос фотолюминесценции в $\mathrm{GaN}$.

вакансиях $\mathrm{O}_{\mathrm{N}}$ с вакансиями галлия $\mathrm{O}_{\mathrm{N}}-V_{\mathrm{Ga}}$ и с гидрогенизированными вакансиями галлия $\mathrm{O}_{\mathrm{N}}-V_{\mathrm{Ga}} \mathrm{H}_{n}$, соответственно, а в области $3.4>h v>3.1$ эВ - с донорноакцепторными парами, также включающими $\mathrm{O}_{\mathrm{N}}$.

Энергетический спектр дефектов в спектральной области $h v>2.2$ эВ можно аппроксимировать экспоненциальной функцией $g\left(E_{t}\right) \sim \exp \left(-E_{t} / E_{0}\right)$ с характеристи- ческой энергией $E_{0}$, зависящей от энергии локализации $E_{t}=E_{c}-E$, отсчитываемой от дна зоны проводимости и имеющей положительное значение. Расстояние от интерфейса $\mathrm{Au} / \mathrm{GaN}$ (от $x=0$ ) на квазиуровне Ферми может быть связано с энергией локализации центров как $x=w-\left[2 \varepsilon_{0} \varepsilon\left(E_{t}(x)\right) / q N_{d}\right]^{1 / 2}[22]$. Здесь $\varepsilon_{0}$ и $\varepsilon-$ электрическая постоянная и относительная диэлектрическая проницаемость соответственно, $w$ - ширина ОП3, $N_{\mathrm{d}}$ - концентрация доноров. По мере того как электрон туннелирует от края ОПЗ в глубь обедненной области, перескакивая в состояния с большей энергией локализации, плотность состояний на транспортном уровне падает, расстояние между ближайшими состояниями увеличивается, и вероятность горизонтальных по энергии прыжков между ними уменьшается. Стационарный ток контролируется плотностью состояний вблизи интерфейса $\mathrm{Au} / \mathrm{GaN} g_{0 b}\left(E_{0 t}\right)$ с энергией локализации $E_{0 t}=q \varphi_{0 b}-\Delta F_{n}$, пересекаемых квазиуровнем Ферми $F_{n}$ при прямом смещении $V_{j}=\Delta F_{n} / q$.

При поглощении фотонов с энергией $h v$ в донорноакцепторных комплексах электроны совершают переход из заполненных акцепторных состояний комплекса $E_{1}$, в пустые донорные энергетические состояния комплекса $E_{2}$. Оптическая плотность $\alpha d(h v)$ определяется плотностью пустых состояний $E_{2}$, как и величина прямого тока через ОПЗ.

При высоте барьера Шоттки $q \varphi_{0 b}=1.12$ эВ, равновесный уровень Ферми на интерфейсе $\mathrm{Au} / \mathrm{GaN}, F_{0 b}$, расположен выше валентной зоны на $F_{0 b}-E_{v}=2.3$ эВ (ширина запрещенной зоны $\mathrm{GaN}$ при $300 \mathrm{~K}, E_{g, \mathrm{GaN}}=3.42$ эВ).

Голубая фотолюминесценция $\left(h v_{\mathrm{BL}}=2.9\right.$ эВ $)$ обусловлена излучательными переходами в донорно-акцепторных комплексах $\mathrm{O}_{\mathrm{N}}-V_{\mathrm{Ga}} \mathrm{H}_{n}$, акцепторные энергетические уровни которого расположены вблизи валентной зоны [17]. Учитывая также стоксов сдвиг между полосами поглощения и фотолюминесценции, можно считать, что при поглощении фотонов с энергией $h v \geq 2.9$ эВ электроны совершают переход из состояний, близких к валентной зоне, в донорные состояния комплекса с энергией $E=E_{v}+h v$, расположенные по энергии на интерфейсе $\mathrm{Au} / \mathrm{GaN}$ выше равновесного уровня Ферми на $E-F_{0 b} \geq 0.6$ эВ. Это позволяет связать рост тока при напряжении $0.9>V_{j}>V_{\text {th }}=0.6 \mathrm{~B}$ (рис. $\left.1, a\right)$ с ростом плотности состояний на квазиуровне Ферми вблизи интерфейса $\mathrm{Au} / \mathrm{GaN}$, ответственных за голубую полосу фотолюминесценции, а рост тока при $V_{j}>0.9-1 \mathrm{~B}$ и $0.6>V_{j}>0 \mathrm{~B}$ - с ростом плотности состояний, ответственных за УФ и желтую фотолюминесценцию соответственно.

Близость квазиуровней Ферми $F_{0 b}=2.3+V_{j}$, при которых наблюдается резкое увеличение проводимости при пороговых напряжениях $V_{\text {th }}=0,0.6$ и $0.9-1 \mathrm{~B}$, равных $F_{0 b}=2.3,2.9$ и $3.2-3.3$ эВ, к оптическим пороговым энергиям $h v_{\text {th }}=2.3,2.9$ и 3.2 эВ, отражающим рост плотности состояний, подтверждает прыжковый механизм прохождения тока. 


\section{4. Обсуждение результатов}

\section{1. Локальная прыжковая проводимость в области пространственного заряда}

Модель дисперсионного переноса в полупроводниках с высокой плотностью локализованных состояний в запрещенной зоне основана на времяпролетных экспериментах (time-of-flight method) [23,24]. В этой модели рекомбинационный ток создается электронами, захваченными на глубокие состояния и продолжающими прыжковое движение по локализованным состояниям до рекомбинации с дырками. Транспорт с участием ловушек в ОПЗ можно рассматривать на основе модели дисперсионного транспорта [23,24]: электроны на границе нейтральной области с ОПЗ совершают горизонтальный по энергии туннельный переход в ближайшую ловушку, а более удаленных достигают путем прыжкового движения по ловушкам.

Предположим простейшую форму частоты перескоков электронов между двумя центрами $i$ и $j$ хвоста зоны проводимости, разделенными расстоянием $R_{i j}$ [23]:

$$
v\left(R_{i j}\right)=v_{0} \exp \left(-2 \gamma R_{i j}\right),
$$

где $v_{0} \approx 10^{12} \mathrm{c}^{-1}$ - частота попыток, $\gamma=1 / a-$ степень перекрытия волновых функций, $a$ - боровский радиус. Вблизи зоны проводимости энергетическое распределение плотности локализованных состояний может быть аппроксимировано экспоненциальной функцией $g\left(E_{t}\right)=\left(N_{t} / E_{\mathrm{U}}\right) \exp \left(-E_{t} / E_{\mathrm{U}}\right)$ с урбаховской энергией $E_{\mathrm{U}}=0.05$ эВ [18]. Здесь $N_{t}-$ полное число локализованных состояний. Учитывая, что $R_{i j}=\left[N_{t}^{-1 / 3} \exp \left(E_{t} / 3 E_{\mathrm{U}}\right)\right]$, для частоты туннельных прыжков электронов на транспортном уровне получим:

$$
v_{\text {hop }}\left(E_{t}\right)=v_{0} \exp \left[-2 \gamma N_{t}^{-1 / 3} \exp \left(E_{t} / 3 E_{\mathrm{U}}\right)\right] .
$$

Локальная прыжковая проводимость в ОПЗ может быть записана в виде

$$
\sigma_{\text {hop }}=q \mu N_{t}(E)\left(k T / E_{\mathrm{U}}\right),
$$

где $\mu=q D / k T-$ эффективная микроскопическая подвижность, $D=R_{i j}^{2} v_{\text {hop }}\left(E_{t}\right)-$ эффективный микроскопический коэффициент диффузии. Окончательно получаем:

$$
\sigma_{\text {hop }}=q^{2} g\left(E_{t}\right) R_{i j}^{2} v_{\text {hop }} .
$$

Так как полосы желтой и голубой фотолюминесценции в $\mathrm{GaN}$ имеют гауссову форму [17], распределение по энергии локализованных состояний, ответственных за оптическое поглощение, $g\left(E_{t}\right)=N_{\mathrm{L}} \rho\left(E_{t}\right)$ может быть также представлено в виде функции Гаусса:

$$
\rho\left(E_{t}\right)=\frac{2}{\sigma \sqrt{\pi}} \exp \left(\frac{\left(E_{t}-E_{0 t}\right)^{2}}{2 \sigma^{2}}\right),
$$

где $\sigma=w_{\mathrm{L}} / 2 \sqrt{\ln 4}\left(w_{\mathrm{L}}\right.$ - полная ширина на половине высоты $(F W H M)$ полосы фотолюминесценции, $E_{0 t}-$

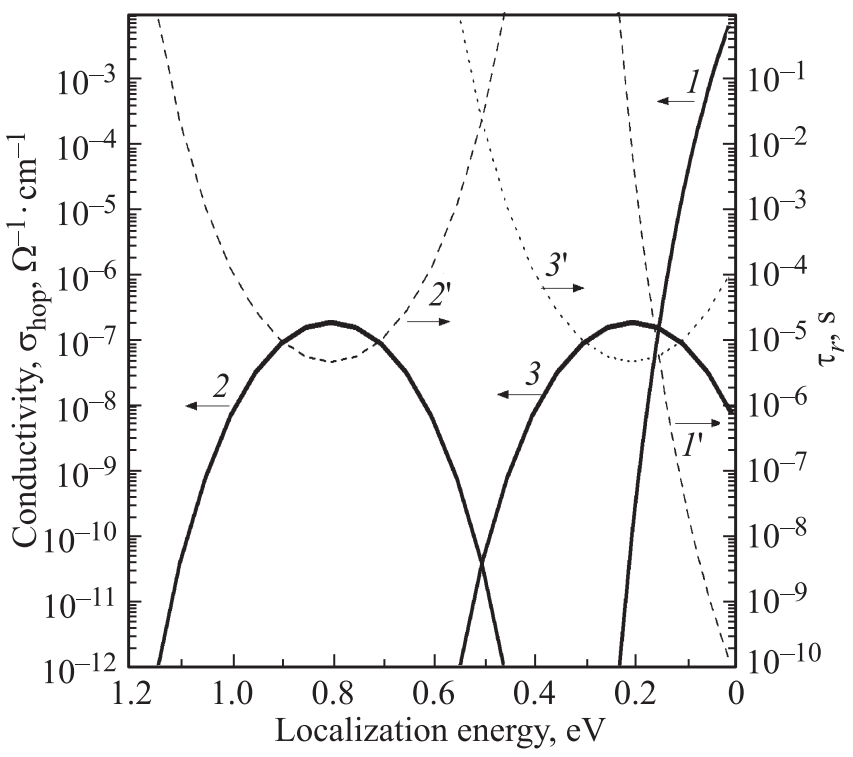

Рис. 3. Расчетные зависимости локальной прыжковой проводимости $(1-3)$ и времени диэлектрической релаксации $\left(1^{\prime}-3^{\prime}\right)$ в области пространственного заряда барьеров Шоттки от энергии локализации для экспоненциального хвоста состояний зоны проводимости $\mathrm{GaN}\left(1,1^{\prime}\right)$ и состояний, ответственных за желтую $\left(2,2^{\prime}\right)$ и голубую $\left(3,3^{\prime}\right)$ фотолюминесценцию. Плотность состояний, $\mathrm{cm}^{-3}: 1,1^{\prime}-2.3 \cdot 10^{18} ; 2,3,2^{\prime}$, $3^{\prime}-10^{17}$.

положение максимума гауссиана, соответствующее энергии фотона в максимуме оптического поглощения, $N_{\mathrm{L}}-$ полное число состояний).

Расчетная зависимость $\sigma_{\text {hop }}\left(E_{t}\right)$ для экспоненциального хвоста зоны проводимости представлена на рис. 3, (кривая 1). Здесь принято, что распределение плотности состояний в зоне проводимости $N_{c}(E)$ плавно переходит в распределение плотности состояний хвоста $N_{t}\left(E_{t}\right)$ [24] и $N_{t}=N_{c}=2.3 \cdot 10^{18} \mathrm{~cm}^{-3}, E_{\mathrm{U}}=0.05$ эВ [18].

Расчетная зависимость $\sigma_{\text {hop }}\left(E_{t}\right)$ для центров, ответственных за желтую фотолюминесценцию, представлена на рис. 3, кривая 2. Зависимость $\sigma_{\text {hop }}\left(E_{t}\right)$ рассчитана при следующих значениях параметров: $N_{\mathrm{L}}=10^{17} \mathrm{~cm}^{-3}$, $a=2.5 \mathrm{Hм}$ (принимая эффективную массу электрона равной $0.2 m_{0}, m_{0}$ - масса свободного электрона), $w_{\mathrm{L}}=0.4$ эВ [17], $h v_{\mathrm{YL}}=2.2$ эВ, $E_{0 t}=0.8$ эВ (положение максимума гауссиана, соответствующее энергии фотона в максимуме оптического поглощения для желтой полосы YL $h v=2.6$ эВ [12]).

Как видно из рис. 3, вблизи края ОПЗ, где $0<E_{t}<0.15$ эВ, присутствует область с высокой локальной прыжковой проводимостью $1>\sigma_{\text {hop }}>10^{-7} \mathrm{OM}^{-1} \mathrm{~cm}^{-1}$ (кривая 1). Вблизи интерфейса $\mathrm{Au} / \mathrm{GaN}$, где $1.12>E_{t}>0.7$ э, присутствует область с низкой проводимостью, для $N_{\mathrm{L}}=10^{17} \mathrm{~cm}^{-3}$ еe проводимость $2 \cdot 10^{-7}>\sigma_{\text {hop }}>10^{-11} \mathrm{OM}^{-1} \mathrm{~cm}^{-1}$ (кривая 2). Здесь предполагается, что при $0.15<E_{t}<0.7$ эВ проводимость $\quad \sigma_{\text {hop }} \geq 10^{-7} \mathrm{OM}^{-1} \mathrm{~cm}^{-1} \quad$ обусловлена 
прыжками между состояниями, ответственными за голубую фотолюминесценцию (кривая 3). С увеличением прямого смещения область с высокой проводимостью приближается к интерфейсу $\mathrm{Au} / \mathrm{GaN}$, а область с низкой проводимостью сужается. Уже при малых смещениях $V_{j}=0-0.3$ В вблизи интерфейса $\mathrm{Au} / \mathrm{GaN}$

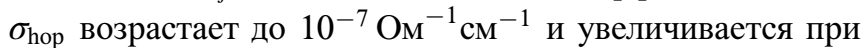
смещениях $V_{j}=1-1.12$ В до $10^{-5}-10^{-2} \mathrm{OM}^{-1} \mathrm{~cm}^{-1}$.

Для диода $\mathrm{C}$ величина проводимости $G_{\mathrm{dc}}$, определенная из $j-V_{j}$-кривых (рис. $1, a$, кривая 3) при $V_{j}=0.3$ В равна $G_{\mathrm{dc}}=5 \cdot 10^{-7} \mathrm{OM}^{-1}$ и при $w=10^{-5} \mathrm{~cm}$ и $S=10^{-4} \mathrm{~cm}^{2}$ находится в согласии с расчетной величиной $\sigma_{\text {hор }}$ для $N_{\mathrm{L}}=10^{17} \mathrm{~cm}^{-3}$, полученной в предположении однородного распределения по площади плотности глубоких центров и плотности тока. В то же время докальная проводимость экспоненциально зависит от плотности центров, и отличие в величине проводимости исследованных диодов, достигающее при малых напряжениях четырех порядков, вероятно связано с более высокой локальной плотностью глубоких центров вблизи дислокаций, границ зерен и других протяженных дефектов. Так, оценки величин $\tau_{\text {hop }}$ и $\sigma_{\text {hop }}$ по формулам (1) и (3) показывают, что локальное повышение плотности центров в 2 раза сопровождается увеличением на порядок частоты перескоков и проводимости. Кроме того, высокая проводимость $G_{\mathrm{dc}}$ диодов А и В (рис. 1, $a$, кривые 1 и 2) может быть связана с высокой туннельной прозрачностью барьера Шоттки, на которую, помимо уровня легирования, может оказывать влияние высокая концентрация непреднамеренно вводимых водородсодержащих донорных центров и вызванное их ионизацией уменьшение ширины ОПЗ [25,2].

Прыжковая проводимость в ОПЗ приводит к изменению распределения потенциала в ОПЗ, определяющегося распределением локальных сопротивлений $V(x) \sim r_{\text {hop }}(x) \sim 1 / \sigma_{\text {hop }}(x)$. На вставке к рис. 1 показано распределение потенциала в ОПЗ при приложении прямого напряжения. Основная часть приложенного напряжения падает вблизи интерфейса $\mathrm{Au} / \mathrm{GaN}$, в области с малой проводимостью $\sigma_{\text {hор }}(x)$ вблизи $x=0$. Вблизи края ОП3, в области с большой проводимостью $\sigma_{\text {hор }}(x)$ вблизи $x=w$, падение напряжения мало и распределение потенциала слабо меняется с напряжением, так что при увеличении напряжения вершина барьера удаляется от поверхности. Высота барьера относительно слабо изменяется в ростом напряжения, но ширина ОПЗ уменьшается, что способствует „проколу“ барьера с ростом прямого напряжения.

\section{2. Локальное время диэлектрической релаксации и переходной прыжковый ток}

Прыжковая природа транспорта в ОПЗ барьеров Шоттки определяет характер переходного и динамического электрического отклика диода при переключениях напряжения и на переменном напряжении, при емкостных $C-V$-измерениях.
На рис. 3 представлены расчетные зависимости локального времени диэлектрической (максвелловской) релаксации $\tau_{r}\left(E_{t}\right)=\varepsilon_{0} \varepsilon / \sigma_{\text {hop }}$ от энергии локализации для состояний хвоста зоны проводимости (кривая $1^{\prime}$ ) и центров, ответственных за желтую и голубую фотолюминесценцию (кривые $2^{\prime}, 3^{\prime}$ ). Зависимости рассчитаны при тех же значениях параметров, что и зависимости $\sigma_{\text {hop }}\left(E_{t}\right)$ (кривые $\left.1-3\right)$.

Как видно из рис. 3, время диэлектрической релаксации в ОПЗ $\tau_{r}$ превышает максвелловское время релаксации в нейтральной области $\mathrm{GaN}$ $\tau_{\mathrm{M}} \sim 10^{-12}-10^{-13}$ с. Замедленная диэлектрическая релаксация в ОПЗ характеризуется широким, охватывающим много порядков, распределением локальных времен $\tau_{r}(x)=\varepsilon_{0} \varepsilon / \sigma_{\text {hop }}(x)$. Частота перескоков уменьшается с расстоянием $w-x=\left(2 \varepsilon_{0} \varepsilon E_{t} / q N_{d}\right)^{1 / 2}$ от края ОП3. Вблизи края ОПЗ, где $E_{t}<0.1 э \mathrm{~B}$, локальное время $\tau_{r}=10^{-7}-10^{-10} \mathrm{c}$, и возрастает от $10^{-7}$ до $10^{-2}$ с вблизи интерфейса $\mathrm{Au} / \mathrm{GaN}$. Замедление скорости прыжкового переноса электронов должно приводить к растянутому во времени переходному прыжковому току.

Величину стационарного тока через ОПЗ ограничивает высокое локальное сопротивление вблизи интерфейса $\mathrm{Au} / \mathrm{GaN}$. Величина переходного тока в момент времени $t$ определяется локальным сопротивлением $r_{\text {hop }}\left(E_{t}\right) \tau_{\text {hop }}\left(E_{t}\right) /\left(q^{2} g\left(E_{t}\right) R_{i j}^{2}\left(E_{t}\right)\right)$. Если мы пренебрежем относительно слабой зависимостью произведения $g\left(E_{t}\right) R_{i j}\left(E_{t}\right) \sim E_{t}^{1 / 3}$ от энергии локализации по сравнению с зависимостью $\sigma_{\mathrm{hop}}\left(E_{t}\right)$, то получим линейную зависимость локального переходного сопротивления от времени перескока $r_{\text {hop }}\left(E_{t, x}\right) \sim \tau_{\text {hop }}\left(E_{t}\right)$. Так как лимитирующим величину переходного тока процессом являются прыжки из состояний, которые имеют характерное время перескока $\tau_{\text {hop }}(x)=t$, переходной ток должен затухать со временем примерно как $I(t) \sim 1 / t$. Отметим, что эта модель дает объяснение временны́м зависимостям переходного тока в GaN-светодиодах [26].

\section{3. Прыжковая емкость области пространственного заряда}

При приложении малого переменного напряжения круговой частоты $\omega<1 / \tau_{\mathrm{M}}$ область у края ОПЗ (вблизи $x=w)$, где $\omega \tau_{r}<1$ и носители успевают следовать за осциллирующим напряжением, вносит вклад в измеряемую дифференциальную активную проводимость $G_{\text {ac }}(\omega)$. Так как по мере удаления от края ОПЗ скорость перескоков из состояний $E_{t}$ меньше скорости перескоков в эти состояния, возникают осцилляции прыжкового тока, по фазе опережающие напряжение. Но одновременно благодаря осцилляциям квазиуровня Ферми и заполнения захватывающих центров инжектированными в ОПЗ электронами инерционно осциллирует и проводимость через ОПЗ, что вызывает осцилляции прыжкового тока, по фазе отстающие от напряжения. При малых прямых смещениях сквозной ток ограничивается малой плотностью состояний $N_{\mathrm{L}}\left(E_{t_{0}}\right)$ на квазиуровне Ферми вблизи 
интерфейса $\mathrm{Au} / \mathrm{GaN}$ и доминирует захват электронов, вызывающий емкостной отклик прыжкового тока и проявляющийся как дифференциальная прыжковая емкость

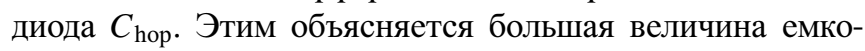
сти диода С при малых $V_{j}$ по сравнению с диодами А и В (рис. $1, b)$. Увеличение плотности состояний $g\left(E_{t}\right)$ на квазиуровне Ферми с ростом смещения приводит к росту величины $G_{\mathrm{dc}}$ и $G_{\mathrm{ac}}$. Когда при увеличении $V_{j}$ $G_{\mathrm{dc}}$ становится больше $\omega C$, осцилляции заряда вблизи квазиуровня Ферми приводят к преимущественному росту проводимости и индуктивный отклик начинает преобладать, проявляясь как отрицательная прыжковая емкость диода.

Индуктивный отклик в диодах $\mathrm{C}$ и В начинает преобладать вблизи $V_{\mathrm{th}}=1 \mathrm{~B}$, в диоде А вблизи $V_{\mathrm{th}}=0.8 \mathrm{~B}$, но ступенчатый рост отрицательной емкости и активной проводимости виден уже при $V_{\mathrm{th}}=0.4$ и $0.6 \mathrm{~B}$ (рис. $1, b$ ). Таким образом, ступенчатый рост $G_{\mathrm{dc}}, \omega C$ и $G_{\mathrm{ac}}$ с $V_{j}$ наблюдается при одних и тех же пороговых напряжениях и связан с увеличением плотности состояний на квазиуровне Ферми. Можно сделать вывод, что зависимости емкости и активной проводимости диодов, так же как и зависимости статической проводимости, отражают энергетическое распределение дефектных состояний в запрещенной зоне $\mathrm{GaN}$.

Как показали $C-V$-измерения на частотах $10-100$ кГц, понижение частоты $\omega$ приводит к уменьшению измеряемой активной проводимости и увеличению измеряемой емкости. Подобный характер изменения емкости и проводимости с частотой напряжения наблюдался и в $\mathrm{GaN}-$-ветодиодах [27]. Частотные зависимости $G_{\mathrm{ac}}(\omega)$ и $C(\omega)$ обусловлены тем, что с понижением частоты электроны проникают за полупериод напряжения глубже в ОП3, и область, где $\tau_{r}<1 / \omega$, расширяется, приближаясь к интерфейсу $\mathrm{Au} / \mathrm{GaN}$, но

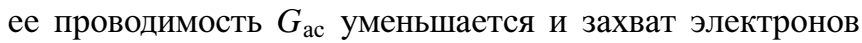
доминирует, приводя к увеличению прыжковой емкости.

Необходимо подчеркнуть, что в отличие от барьерной емкости, величина прыжковой емкости $C_{\text {hop }}$, при одинаковых амплитудах осцилляций тока одинакова как при равномерной по площади плотности тока, так и при локализации тока в отдельных участках, например, при утечках тока вдоль дислокаций или по боковой поверхности. При этом зависимость $C(V)$ определяется энергетическим распределением локализованных состояний в запрещенной зоне, а не пространственным профилем концентрации примеси, как в случае барьерной емкости.

Детальный анализ динамического АС-отклика области объемного заряда барьера Шоттки для полупроводника с высокой и непрерывной плотностью глубоких состояний $[28,29]$ основывался на процессе термоактивации носителей из локализованных состояний в зону. В то же время в работах $[28,29]$ отмечалось, что при высокой плотности состояний прямой прыжковый перенос из обедненной области может быть более быстрым, чем термическая активация. Результаты емкостных измерений в $\mathrm{GaN}$ анализируются в литературе на основе термоактивационного механизма. При измерениях методом релаксационной емкостной спектроскопии (DLTS) такая интерпретация экспериментальных данных обычно приводит к величине плотности глубоких центров $\sim 10^{13}-10^{14} \mathrm{~cm}^{-3}$. При измерениях емкости на частоте 1 МГц, в микросекундном временно́м диапазоне, и измерениях переходной емкости после переключения напряжения в миллисекундном „окне скоростей“ („rate windows") относительное изменение емкости составляет обычно около $0.1 \%$, что приравнивается к отношению плотности глубоких и мелких примесей. Однако прыжковый ток носителей из глубоких центров в ОПЗ уменьшается после переключения напряжения со временем как $1 / t$ (см. разд. 4.1) и в миллисекундном временно́м диапазоне составляет лишь $\sim 10^{-3}$ от начального тока захваченных в ОПЗ носителей. Соответственно плотность глубоких центров в $\sim 10^{3}$ раз больше, чем полученная в рамках модели термического выброса захваченных носителей в зону, и составляет по крайней мере $10^{16}-10^{17} \mathrm{~cm}^{-3}$ при относительном изменении емкости $0.1 \%$. Высокая энергетическая плотность состояний в хвостах зон подтверждается большим коэффицентом подзонного оптического поглощения в $\mathrm{GaN}$, достигающего в видимой и ближней УФ областях $10^{2}-10^{3} \mathrm{~cm}^{-1}[19]$. Оценка нижнего предела концентрации центров поглощения по известной формуле Смакулы-Декстера [30]:

$$
N_{\mathrm{L}}=0.87 \cdot 10^{17} \cdot \alpha w_{\mathrm{L}} n_{\lambda} /\left(f\left(n_{\lambda}^{2}+2\right)^{2}\right),
$$

где $n_{\lambda}$ - коэффициент преломления для длины волны, соответствующей максимуму полосы поглощения, $f$ сила осциллятора, $\alpha-$ коэффициент поглощения в максимуме полосы $\left(\mathrm{cm}^{-1}\right)$, для полосы поглощения с максимумом при $h v=2.6$ эВ, с использованием следующих значений параметров: $n_{\lambda}=2.5, f=1, w_{\mathrm{L}}=0.4$ эВ и $\alpha=10^{2}$, дает значение порядка $N_{\mathrm{L}}=10^{17} \mathrm{~cm}^{-3}$.

\section{5. Заключение}

Экспоненциальное распределение плотности состояний дефектов в $\mathrm{GaN}$ и их высокая плотность приводят к возникновению прыжковой проводимости через область пространственного заряда, кардинально изменяя распределение электрического поля в области пространственного заряда, время диэлектрической релаксации и вид $I-V$ и $C-V$ характеристик диода Шоттки. Характер переходного и динамического отклика диода при прямом смещении определяется конкуренцией двух процессов. Уменьшение плотности состояний и замедление скорости горизонтального по энергии прыжкового переноса электронов при движении к интерфейсу $\mathrm{Au} / \mathrm{GaN}$ вызывает уменьшение прыжкового тока и емкостной отклик диода. В то же время вертикальное по энергии перемещение квазиуровня Ферми сопровождается инерционным возрастанием прыжковой проводимости 
и индуктивным откликом диода (отрицательной емкостью). При этом зависимости тока и емкости от прямого смещения диодов Шоттки на $n-\mathrm{GaN}$ отражают энергетическое распределение плотности состояний дефектов в запрещенной зоне.

\section{Список литературы}

[1] A. Lidow, J. Strydom, M. Rooij, D. Reusch. GaN transistors for efficient power conversion (Chichester, Wiley, 2015).

[2] Z. Yatabe, J.T. Asubar, T. Hashizume. J. Phys. D: Appl. Phys., 49, 393001 (2016).

[3] M.E. Levinshtein, S.L. Rumyantsev, R. Gaska, J.W. Yang, M.S. Shur. Appl. Phys. Lett., 73, 1089 (1998).

[4] L.S. Yu, Q.Z. Liu, Q.J. Xing, D.J. Qiao, S.S. Lau, J. Redwing. J. Appl. Phys., 84, 2099 (1998).

[5] K. Suzue, S.N. Mohammad, Z.F. Fan, W. Kim, O. Aktas, A.E. Botchkarev, H. Morkoç. J. Appl. Phys., 80, 4467 (1996).

[6] D. Yan, J. Jiao, J. Ren, G. Yang, X. Gu. J. Appl. Phys., 114, 144511 (2013).

[7] S. Karmalkar, D.M. Sathaiya, M.S. Shur. Appl. Phys. Lett., 82, 3976 (2003).

[8] J. Racko, J. Pecháček, M. Mikolášek, P. Benko, A. Grmanová, L. Harmatha, J. Breza. Radioengineering, 21, 213 (2012).

[9] С.Д. Ганичев, И.Н. Яссиевич, В. Преттл. ФТТ, 39, 1905 (1997).

[10] E.J. Miller, E.T. Yu, P. Waltereit, J.S. Speck. Appl. Phys. Lett., 84, 535 (2004).

[11] T. Sawada, Y. Ito, K. Imai, K. Suzuki, H. Tomozawa, S. Sakai. Appl. Surf. Sci., 159-60, 449 (2000).

[12] Н.И. Бочкарева, И.А. Шеремет, Ю.Г. Шретер. ФТП, 50, 1387 (2016).

[13] V. Voronenkov, N. Bochkareva, R. Gorbunov, P. Latyshev, Y. Lelikov, Y. Rebane, A. Tsyuk, A. Zubrilov, Y. Shreter. Jpn. J. Appl. Phys., 52, 08JE14 (2013).

[14] F. Ioculano. F. Roccaforte, F. Giannazzo, V. Raineri. Appl. Phys. Lett., 90, 092119 (2007).

[15] M. Mamor. J. Phys.: Condens. Matter, 21, 335802 (2009).

[16] T. Mori, T. Kozawa, T. Ohwaki, Y. Taga, S. Nagai, S. Yamasaki, S. Asami, N. Shibata, M. Koike. Appl. Phys. Lett., 69, 3537 (1996).

[17] M.A. Reshchikov, H. Morkoç. J. Appl. Phys., 97, 061301 (2005).

[18] C.H. Qiu, C. Hoggatt, W. Melton, M.W. Leksono, J.I. Pankove. Appl. Phys. Lett., 66, 2712 (1995).

[19] O. Ambacher, W. Reiger, P. Ansmann, H. Angerer, T.D. Moustakas, M. Stutzmann. Sol. St. Commun., 97, 365 (1996).

[20] L. Balagurov, P.J. Chong. Appl. Phys. Lett., 68, 43 (1996).

[21] P.B. Klein, S.C. Binari. J. Phys.: Condens. Matter, 15, R1641 (2003).

[22] S.M. Sze. Physics of Semiconductor Devices, 2nd ed. (Wiley, N. Y., 1981).

[23] D. Monroe. Phys. Rev. Lett., 54, 146 (1985).

[24] T. Tiedje, A. Rose. Sol. St. Commun., 37, 49 (1980).

[25] Н.И. Бочкарева, Ю.Т. Ребане, Ю.Г. Шретер. ФТП, 49, 1714 (2015).

[26] Н.И. Бочкарева, Е.А. Zhirnov, А.А. Ефремов, Ю.Т. Ребане, Р.И. Горбунов, Ю.Г. Шретер. ФТП, 39, 627 (2005).

[27] Н.И. Бочкарева, В.В. Вороненков, Р.И. Горбунов, Ф.Е. Латышев, Ю.С. Леликов, Ю.Т. Ребане, А.И. Цюк, Ю.Г. Шретер. ФТП, 47, 129 (2013).
[28] D.V. Lang, J.D. Cohen, J.P. Harbison. Phys. Rev. B, 25, 5285 (1982).

[29] J.D. Cohen, D.V. Lang. Phys. Rev. B, 25, 5321 (1982).

[30] D.L. Dexter. Phys. Rev., 101B, 48 (1956).

Редактор А.Н. Смирнов

\section{Hopping conduction and dielectric relaxation in Schottky barriers on GaN}

N.I. Bochkareva ${ }^{1}$, V.V. Voronenkov ${ }^{1}$, R.I. Gorbunov ${ }^{1}$, M.V. Virko ${ }^{2}$, V.S. Kogotkov ${ }^{2}$, A.A. Leonidov', P.N. Vorontsov-Velyaminov ${ }^{3}$, I.A. Sheremet ${ }^{4}$, Y.G. Shreter ${ }^{1}$

${ }^{1}$ loffe Institute, 194021 St. Petersburg, Russia

${ }^{2}$ Peter the Great St. Petersburg Polytechnic University, 195251 St. Petersburg, Russia

${ }^{3}$ Saint Petersburg State University,

199034 St. Petersburg, Russia

${ }^{4}$ Financial University under the Government of the Russian Federation, 125993 Moscow, Russia

Abstract We present results of the study on dependences of current and capacitance on the forward voltage in $\mathrm{Au} / n-\mathrm{GaN}$ Schottky diodes, sub-band optical absorption and defect photoluminescence in $n$-GaN bulk crystals and thin layers of $n$-GaN. It is shown that the defect-assisted tunneling is the dominant transport mechanism at the forward biases of Schottky contacts on GaN. The dependences of current and capacitance on the forward junction voltage reflect the energy spectrum of defects in the energy gap of $n$-GaN: the increase with energy of density of deep states responsible for yellow photoluminescence in $\mathrm{GaN}$ and the steep exponential tail of states with the Urbach energy of $E_{\mathrm{U}}=50 \mathrm{meV}$ near the conduction band edge. The decrease of frequency of electron hops in the region near $\mathrm{Au} / n-\mathrm{GaN}$ interface results in a wide distribution of local dielectric relaxation times and dramatic transformation of the electric field in the space-charge-region at forward voltages. 